\title{
Bürgerschaftliches Engagement für und von Familien - Eine empirische Analyse mit dem Freiwilligensurvey 1999-2009
}

\author{
Volunteer services provided for and by families: An empirical analysis \\ with data from the German "Freiwilligensurvey" 1999-2009
}

\begin{abstract}
Zusammenfassung:
Der Beitrag beschäftigt sich mit der Rolle von bürgerschaftlichem Engagement, das für Familien erbracht wird und das Familien selbst erbringen. Dabei werden unter bürgerschaftlichem Engagement für und von Familien Betreuungs-, Beratungs- und Pflegeleistungen für Kinder, Jugendliche oder Senioren betrachtet, die Familien auf direkte oder indirekte Weise entlasten. Die Daten des Freiwilligensurvey zeigen, dass 35 Prozent der bürgerschaftlich engagierten Menschen in Deutschland ihre Tätigkeit direkt oder indirekt an Familien ausrichten. Familienmitglieder gehören zugleich zu den Hauptakteuren bürgerschaftlicher Arbeit. Insbesondere in Haushaltskontexten mit Kindern sind mehr als 40 Prozent aller Engagierten für andere Familien aktiv. Vor dem Hintergrund aktueller Problemlagen von Familien und des demografischen Wandels kann in Zukunft mit einem deutlich steigenden Bedarf an bürgerschaftlicher Unterstützung gerechnet werden.
\end{abstract}

Schlagwörter: bürgerschaftliches Engagement, Problemlagen von Familien, Pflege und Betreuung, Freiwilligensurvey, demografischer Wandel

\begin{abstract}
:
The article deals with the role of volunteer services provided for and by families. This includes care and consulting services for children, adolescents and seniors and is supposed to disburden families in a direct or indirect way. Data from the German "Freiwilligensurvey" (Volunteer Survey) suggest that a proportion of 35 percent of all volunteers aim their services at families. On the other side, families belong to the main actors of volunteering. In household with children, more than 40 percent of the volunteers are engaged for other families. Against the background of current problems in family life and demographic change, a raise in demand for volunteer services is likely for the future.
\end{abstract}

Key words: volunteer services, problems in family-life, care services, German Volunteer Survey, demographic change

1 Bedanken möchten wir uns bei Thomas Gensicke (TNS Infratest) für viele methodische Erläuterungen sowie bei den drei anonymen Gutachtern, für deren konstruktive Hinweise. 


\section{Einleitung}

Bürgerschaftliches Engagement für und von Familien ist bisher nur selten Gegenstand einer eigenständigen Untersuchung gewesen (Klocke/Limmer/Lück 2001; Klocke/Stadtmüller 2009; Alscher/Dathe/Priller/Speth 2009), obwohl ein Großteil bürgerschaftlichen Engagements von Familienmitgliedern in Anspruch genommen bzw. von Familien erbracht wird. So kommt die Studie von Klocke, Limmer und Lück auf Basis des Freiwilligensurveys 1999 zu der Einschätzung, dass etwa 40 Prozent des gesamten bürgerschaftlichen Engagements in Deutschland jungen Familien, das heißt Familien mit minderjährigen Kindern im Haushalt, zugute kommt (ähnlich Klocke/Stadtmüller 2009). Dabei handelt es sich vorzugsweise um Unterstützungen im Bereich Erziehung und Bildung. Insgesamt wird deutlich, dass nahezu alle Familien mit minderjährigen Kindern zumindest phasenweise Unterstützung durch bürgerschaftliches Engagement erfahren. Die Engagierten gehören meist selbst der Gruppe der Familien an (z.B. als Elternvertreter in Kindergärten oder Schulen) bzw. haben eine enge Beziehung zur Lebensform Familie (z.B. Eltern in der Phase des empty nest). Zusammenfassend zeigt sich, dass Familien in erheblichem Umfang von bürgerschaftlichem Engagement profitieren, zugleich aber auch zu den Hauptakteuren bürgerschaftlicher Arbeit gehören.

Die vorliegende Analyse zielt darauf ab, den Stellenwert des bürgerschaftlichen Engagements für und von Familien näher zu beleuchten und im Zeitverlauf von 1999 bis 2009 zu betrachten. Dabei wird die Familie gleichermaßen als Nehmer und Geber bürgerschaftlichen Engagements betrachtet. Als Datengrundlage dienen die drei Wellen des Freiwilligensurvey von 1999, 2004 und 2009, die somit auch erste Aussagen zu Trends bezüglich des bürgerschaftlichen Engagements für und von Familien erlauben. Der Beitrag gliedert sich in vier Teile: Der erste Teil widmet sich der begrifflichen Abgrenzung des bürgerschaftlichen Engagements. Im zweiten Teil werden die beiden Konzepte bürgerschaftliches Engagement und Familie zusammengeführt. Hierzu erfolgt zunächst eine Darlegung des Familienbegriffs und eine Auseinandersetzung mit der Frage, welche Rolle bürgerschaftliches Engagement für Familien - sowohl auf der Nehmer- als auch auf der Geberseite - spielt bzw. vor dem Hintergrund des Wandels der Familien, der Differenzierung der Lebens- und Familienformen und der demografischen Entwicklung zukünftig spielen könnte. Nach einer knappen Vorstellung des zugrunde liegenden Datenmaterials werden ausgewählte empirische Befunde präsentiert (Kapitel 4). Der Beitrag schließt mit einem Ausblick.

\section{Bürgerschaftliches Engagement}

Das bürgerschaftliche Engagement in Deutschland wird, je nach Studie, zwischen 10 und über 40 Prozent der Bevölkerung gemessen, wobei der Freiwilligensurvey 2009 eine Quote von 36 Prozent ausweist. Diese ernormen Schwankungen mögen z.T. mit dem unterschiedlichen Studien- und Stichprobendesign erklärt werden können, sie spiegeln aber auch die Schwierigkeiten in der begrifflichen Abgrenzung des bürgerschaftlichen Engagements selbst wider. Die Eckpfeiler des bürgerschaftlichen Engagementbegriffs, nämlich 
Freiwilligkeit, Öffentlichkeit und Organisationsbindung, schließen bei enger Auslegung einen Teil des bürgerschaftlichen Engagements aus (Priller 2004). So werden viele fluide Formen der Nachbarschaftshilfe nicht gezählt, obwohl sie für die betroffenen Familien bzw. Menschen verlässliche und womöglich auch langfristige Formen der Unterstützung darstellen. Bei sehr weiter Auslegung des bürgerschaftlichen Engagements werden hingegen Tätigkeitsformen erfasst, die nur formal den Charakter der Freiwilligkeit tragen, für den Einzelnen jedoch Erwerbscharakter haben. Mancher Trainer im Sportverein oder einige Lehrbeauftragte an Hochschulen „leben“ in erheblichem Umfang von den Aufwandsentschädigungen bzw. Anerkennungszahlungen (vgl. hierzu auch Corsten/Kauppert 2007: 347f.). Der Freiwilligensurvey grenzt „,freiwilliges Engagement“ zunächst von „Gemeinschaftsaktivität" ab. Gemeinschaftsaktivitäten bezeichnen eine aktive Teilnahme (z.B. im Sportverein) ohne besonderes Engagement. Weiterhin werden „formelle“ wie „informelle" Formen des bürgerschaftlichen Engagements unterschieden (Gensicke 2005a: 33f.). Hierbei bleiben die informellen Formen des bürgerschaftlichen Engagements auf Aktivitäten innerhalb von Organisationen, Gruppen oder Einrichtungen bezogen, in denen kein Amt (Vorstand, Kassenwart o.ä.) ausgeübt wird. Informelle Formen des bürgerschaftlichen Engagements außerhalb solcher Einrichtungen, z.B. Nachbarschaftshilfe, werden hier jedoch nicht betrachtet. ${ }^{2}$ In den folgenden Analysen nehmen wir diese Definition des Freiwilligensurvey zur Grundlage und betrachten ausschließlich freiwillige formelle wie informelle Tätigkeiten innerhalb von Organisationen bzw. Gruppen.

\section{Der Familienbegriff und die Bedeutung bürgerschaftlichen Engagements für und von Familien}

Der aktuellen Ausgabe des Datenreports zufolge zählt das Statistische Bundesamt auf Basis des Mikrozensus für das Jahr 2006 knapp 9 Millionen Familien in Deutschland. Darunter werden ganz allgemein Mehrpersonenhaushalte mit minderjährigen Kindern verstanden (Statistisches Bundesamt 2008: 33). Werden auch erwachsene Kinder im Haushalt berücksichtigt, so erhöht sich die Zahl auf etwa 12 Millionen. Damit ist nur knapp ein Drittel aller Haushalte in Deutschland als Familie anzusehen (Schneider 2008). Jedoch muss berücksichtigt werden, dass in dieser Querschnittsbetrachtung viele Menschen nicht erfasst werden, die zuvor in Familien gelebt haben (heute: empty nest) oder zukünftig eine Familie gründen werden (junge Paare). Innerhalb der Lebensform Familie gibt es wiederum eine ganze Reihe recht unterschiedlicher Familienkonstellationen, die je eigene Unterstützungsbedarfe bzw. Potentiale bürgerschaftlichen Engagements erwarten lassen (z.B.

2 Der Freiwilligensurvey enthält folgende Fragen, die auf informelles Engagement außerhalb von Organisationen und Einrichtungen abzielen: „Wenn Sie mal Hilfe brauchen, z.B. bei Besorgungen, kleineren Arbeiten oder der Betreuung von Kindern oder Kranken: Gibt es da Personen außerhalb Ihres Haushaltes, an die Sie sich ohne Probleme wenden können?" sowie „Gibt es umgekehrt Personen außerhalb Ihres Haushalts, denen Sie selbst regelmäßig oder gelegentlich helfen, z.B. bei Besorgungen, kleineren Arbeiten oder der Betreuung von Kindern oder Kranken?" 87 Prozent der Befragten antworten im Freiwilligensurvey 2009 auf die erste Frage mit ,ja“, davon nennen knapp die Hälfte (48 Prozent) Nachbarn als potenzielle Unterstützer. Zudem antworten 64 Prozent der Befragten auf die zweite Frage mit „Ja“, hiervon nennen 44 Prozent die Nachbarn als Empfänger. 
Einelternfamilien oder Patchworkfamilien) (Schneider 2008; Geiss/Picot 2009). Familie zeichnet sich allgemein durch eine Eltern-Kind Beziehung aus. Das heißt, wir betrachten Konstellationen von Eltern mit ihren eigenen oder sozialen (z.B. adoptierten) Kindern ebenso wie die Beziehungen der Eltern zu ihren eigenen Eltern (den Großeltern in der Dreigenerationenperspektive). Diese Erweiterung der Perspektive, von der Kernfamilie auf die Familienverbände, ist insbesondere bei der Betrachtung von Pflegeleistungen im Familienverband wichtig, da hier Leistungen für die ältere Generation indirekt der Kernfamilie zu Gute kommen können. Wir betrachten im Folgenden alle Formen bürgerlichen Engagements, die von einzelnen Mitgliedern der Familie erbracht werden, als bürgerschaftliches Engagement von Familien. Ebenso werden alle Formen bürgerschaftlichen Engagements, die direkt oder indirekt dem Familiensystem oder einzelnen Familienmitgliedern zu Gute kommen, als Leistungen für Familien gezählt (vgl. auch Klocke/Stadtmüller 2009; Heitkötter/Jurczyk 2009). Auch diese technische Operationalisierung unseres Analysezugangs lässt einige Punkte offen, die nur pragmatisch behandelt werden können. So lassen sich viele Beispiele finden, in denen nicht das Familienleben unmittelbar, wohl aber Menschen außerhalb der familialen Alltagsroutinen durch bürgerschaftliches Engagement Unterstützung erfahren (bspw. in Sportvereinen), was durchaus positive Effekte (Rückwirkungen) auf das Familiensystem selbst haben kann. Das hängt damit zusammen, das die ureigensten Aufgaben und Leistungen der Familie, nämlich: Kohäsion und emotionale Stabilisierung der Familienmitglieder, Pflege und Erziehung der Kinder, Haushaltsführung, Gesundheit und Erholung sowie wechselseitige Hilfe (Kaufmann 1995), nicht ausschließlich innerhalb des Familiensystems selbst herstellbar sind, sondern zu einem Teil der familialen Umwelt entspringen.

Familien sind aber nicht nur Empfänger, sondern auch Lernort und Geber bürgerschaftlichen Engagements. Da ein Großteil bürgerschaftlichen Engagements von Menschen aus Familien erbracht wird (vgl. Kapitel 4 sowie Klocke/Limmer/Lück 2001), stellen Familien einen bevorzugten Lernort für das nachwachsende bürgerschaftliche Engagement dar. Dabei wird bürgerschaftliches Engagement definitionsgemäß außerhalb der eigenen Familie erbracht und muss auch nicht unmittelbar anderen Familien zufließen. Aber ein wichtiger Bereich der Einübung bürgerschaftlichen Engagements stellt zweifellos die freiwillige Arbeit Jugendlicher in Jugendverbänden, Sportvereinen und Hilfsnetzwerken (z.B. Nachhilfe) dar. Inwieweit die Anleitung für dieses bürgerschaftliche Engagement im Familienkreis gelegt wird, lässt sich nicht eruieren. Jedoch kann davon ausgegangen werden, dass viele Engagementformen von den Eltern übernommen werden (vgl. hierzu auch Klocke/Stadtmüller 2010). Dies mag in religiös geprägten Elternhäusern ein jugendliches Engagement in Kirche und Gemeinde hervorbringen ebenso wie Engagementformen bei den Pfadfindern, den Naturfreunden, der Gewerkschaft, einer politischen Partei oder im Sportverein familial vererbt werden. Corsten und Kauppert (2007) beziehen sich in ihrer Analyse der Motivgenese bürgerschaftlichen Engagements letztlich auf Bourdieus Habituskonzept, wenn sie ausführen "Ein Akteur schöpft sein Selbstverständnis aus dem was schon da ist: Sedimente eigener Erfahrungen, gesellschaftliche Erwartungen, sozialhistorisch bedingte Lagerungen." (S. 349). In der Bezugnahme auf Bourdieu ist für bürgerschaftliches Engagement ein hoher Grad an Habitustradierung von der Elternauf die Kindergeneration erwartbar. Insgesamt muss aber konstatiert werden, dass über die Weitergabe bürgerschaftlichen Engagements von der älteren an die jüngere Generati- 
on im Familienverband bisher nur wenige Erkenntnisse (Corsten/Kauppert 2007) vorliegen.

Dass Familien von bürgerschaftlichem Engagement profitieren, zeigt allein schon der große Kranz an unterstützenden Engagementformen im Umfeld von Erziehung und Schule. In welchem Umfang bürgerschaftliches Engagement zur Entlastung der nehmenden Familie beiträgt, ist jedoch unbekannt. Ebenso kann erbrachtes bürgerschaftliches Engagement der einzelnen Familienmitglieder im Umkehrschluss auch zu Belastungen des gebenden Familiensystems führen. Auch hierzu ist die Forschungslage unbefriedigend. Wie sich die bürgerschaftlichen Leistungen für und von Familien in den letzten zehn Jahren konkret entwickelt haben, wird nun in den nächsten Abschnitten dargelegt.

\section{Daten und Ergebnisse}

\subsection{Die Datenbasis}

Die folgenden Auswertungen zum bürgerschaftlichen Engagement für und von Familien basieren auf den drei Erhebungswellen des Freiwilligensurvey aus den Jahren 1999, 2004 und 2009. Diese Studie, vom Bundesministerium für Familie, Senioren, Frauen und Jugend (BMFSFJ) in Auftrag gegeben und von TNS Infratest durchgeführt (vgl. Gensicke 2005a), enthält - nimmt man alle Befragungswellen zusammen - Informationen von knapp 50.000 Personen über die Ausübung sowie die Art und den Umfang von Freiwilligenarbeit, ehrenamtlichen Tätigkeiten und bürgerschaftlichem Engagement. Die zufällige Auswahl der Haushalte und, auf der untersten Ebene, der Befragungspersonen stellt sicher, dass die Erhebung für die Wohnbevölkerung Deutschlands ab 14 Jahren repräsentativ ist. Die Befragung erfolgte per Telefon (Computer Assisted Telephone Interview, CATI) und anhand eines überwiegend standardisierten Fragebogens.

\subsection{Bürgerschaftliches Engagement für Familien}

Blickt man in einem ersten Schritt auf die Engagementquote, so übernehmen im Jahre 2009 35,9 Prozent der Bevölkerung freiwillig und unentgeltlich (oder gegen eine geringe Aufwandsentschädigung) längerfristig Arbeiten, Aufgaben oder Funktionen und gelten somit als freiwillig engagiert. Im Jahr 1999 betrug dieser Anteil noch 34,0 Prozent. Dagegen ist gegenüber der Erhebungswelle von 2004 ein allenfalls marginaler Anstieg der Engagementquote zu erkennen (+ 0,2 Prozent). Das beliebteste Tätigkeitsfeld im Jahre 2009 stellt der Bereich „Sport und Bewegung“ dar (vgl. Tabelle 1): 28,2 Prozent aller bürgerschaftlich Engagierten sind hier aktiv. Auch die Bereiche „Schule und Kindergarten“, „Kirche und Religion“ (jeweils 19,1\%) sowie „Kultur und Musik“ (zu 14,6\%) und der soziale Bereich (14,5\%) weisen hohe Engagementquoten auf.

Wie kann aber nun ermittelt werden, ob die Aktivitäten der freiwillig Engagierten auch Familien zugute kommen? Folgt man weitgehend der Vorgehensweise von Klocke, Limmer und Lück (2001: 53), so gelingt eine Schätzung unter dem Rückgriff auf eine Kombination von zwei Indikatoren: Zum einen das Tätigkeitsfeld des Engagements, das einen Familien- 
bezug mehr oder weniger erwartbar macht, und zum anderen die vom Befragten angegebene Zielgruppe der Tätigkeit. So ist ein hoher Familienbezug zu erwarten, wenn sich die Tätigkeit in den Bereichen „Schule und Kindergarten“ (z.B. Elternbeiräte), „Kultur und Musik“ (Musiklehrer), „Freizeit und Geselligkeit“ (Betreuung von Jugend- oder Kinderfreizeiten), „Kirche und Religion“ (Kindergottesdienst), „Sozialer Bereich“ (Beratung) oder in „außerschulische Jugendarbeit und Erwachsenenbildung“ (Arbeit in Jugendclubs) vollzieht und als Zielgruppen „Kinder und Jugendliche“, „Familien“ oder „Frauen“ genannt werden. ${ }^{3}$ Auf dieser Basis gelangt man zu dem Ergebnis, dass 29,8 Prozent (gegenüber 29,6 Prozent in 2004) der bürgerschaftlich Aktiven für Familien tätig sind. ${ }^{4}$

Bürgerschaftliches Engagement, das Familien unmittelbar zugute kommt, spielt jedoch nur eine untergeordnete Rolle. So nehmen die Engagierten nur in sehr wenigen Fällen (zu 4,3 Prozent, gegenüber 5,1 Prozent in 2004) die Familien als primäre Adressaten ihrer bürgerschaftlichen Aktivität wahr. Dabei stechen besonders die Tätigkeitsbereiche „Freizeit und Geselligkeit“ sowie „Kirche und Religion“ hervor. Personen, die sich in diesen Feldern engagieren, identifizieren zu 7,7 bzw. zu 6,0 Prozent Familien als primäre Zielgruppe.

Tabelle 1: Bürgerschaftliches Engagement nach Tätigkeitsfeldern und Zielgruppenorientierung ${ }^{5}$

\begin{tabular}{|c|c|c|c|c|}
\hline Tätigkeitsbereich & $\begin{array}{l}\text { Anteil an allen } \\
\text { bürgerschaftlich } \\
\text { Engagierten }\end{array}$ & $\begin{array}{l}\text { Zielgruppe: } \\
\text { Kinder und } \\
\text { Jugendliche }\end{array}$ & $\begin{array}{l}\text { Zielgruppe: } \\
\text { ältere } \\
\text { Menschen }\end{array}$ & $\begin{array}{c}\text { Zielgruppe: } \\
\text { Familien }\end{array}$ \\
\hline Sport und Bewegung & $28,2 \%$ & $45,9 \%$ & $7,1 \%$ & $4,3 \%$ \\
\hline Schule und Kindergarten & $19,1 \%$ & $72,7 \%$ & $1,4 \%$ & $4,2 \%$ \\
\hline Kirche und Religion & $19,1 \%$ & $30,9 \%$ & $11,9 \%$ & $6,0 \%$ \\
\hline Sozialer Bereich & $14,6 \%$ & $20,8 \%$ & $28,9 \%$ & $4,8 \%$ \\
\hline Kultur und Musik & $14,5 \%$ & $23,6 \%$ & $7,3 \%$ & $4,5 \%$ \\
\hline Freizeit und Geselligkeit & $12,8 \%$ & $23,9 \%$ & $11,3 \%$ & $7,7 \%$ \\
\hline Unfall-/Rettungsdienst, Feuerwehr & $8,6 \%$ & $21,2 \%$ & $2,7 \%$ & $2,1 \%$ \\
\hline Umwelt-, Natur- und Tierschutz & $7,8 \%$ & $14,0 \%$ & $7,6 \%$ & $4,0 \%$ \\
\hline Politik & $7,5 \%$ & $18,4 \%$ & $6,6 \%$ & $4,3 \%$ \\
\hline Jugendarbeit und Erwachsenenbildung & $7,2 \%$ & $51,5 \%$ & $4,5 \%$ & $3,0 \%$ \\
\hline Gesundheit & $6,0 \%$ & $15,6 \%$ & $26,0 \%$ & $3,0 \%$ \\
\hline
\end{tabular}

Quelle: Freiwilligensurvey 2009 (gewichtete Ergebnisse), eigene Berechnungen

3 Der Bereich der ,außerschulischen Kinder- und Jugendarbeit“ fällt streng genommen in den Leistungsbereich der Jugendhilfe. Wir gehen jedoch davon aus, das diese Leistung von den betroffenen Familien als Entlastung wahrgenommen werden. Dementsprechend zählen wir sie als Engagement für Familien.

4 Klocke, Limmer und Lück (2001: 53) kommen für das Jahr 1999 zu dem Ergebnis, dass der Anteil des familienbezogenen bürgerschaftlichen Engagements rund 40 Prozent beträgt. Für den Unterschied zu den hier berichteten Ergebnissen ist in erster Linie die Tatsache verantwortlich, dass Klocke, Limmer und Lück auch den beliebten Tätigkeitsbereich „Sport und Bewegung“ als familiennah betrachteten. Darüber hinaus bildeten in diesem Bericht die ehrenamtlichen Tätigkeiten (und nicht die ehrenamtlich Tätigen) die Prozentuierungsbasis. Schließlich haben sich die Antwortkategorien des Zielgruppen-Indikators nach 1999 verändert, so dass auch dies z.T. für die Unterschiede verantwortlich sein mag.

5 Aufgrund von Mehrfachnennungen (43 Prozent der ehrenamtlich Aktiven üben mehr als eine Tätigkeit aus) addieren sich die Werte in der ersten Spalte nicht zu 100 Prozent. 
Richtet sich ein bürgerschaftliches Engagement an Kinder und Jugendliche, so kann - in Abhängigkeit vom Engagementbereich - ein mittelbarer Familienbezug angenommen werden. 34 Prozent der bürgerschaftlich Aktiven geben diese beiden Gruppen als Zielgruppen ihres Engagements an. Besonders hoch ist die Orientierung an Kindern und Jugendlichen naturgemäß in den Tätigkeitsfeldern „Schule und Kindergarten“ (zu 72,7 Prozent) und „Jugendarbeit und Erwachsenenbildung“ (zu 51,5 Prozent).

Eine mittelbare Entlastung von Familien kann nicht nur unterstellt werden, wenn sich bürgerschaftliches Engagement an der Zielgruppe der Kinder und Jugendlichen orientiert, sondern vielfach auch dann, wenn pflegebedürftige ältere Menschen im Zentrum des Engagements stehen. Um das Ausmaß an bürgerschaftlichem Engagement zu quantifizieren, das Familien vermittelt über die Betreuung von pflegebedürftigen Personen zugute kommt, wird wiederum auf die von der Befragungsperson identifizierte Zielgruppe (hier: Ältere) und das Tätigkeitsfeld des Engagements zurückgegriffen.

Zunächst ist ein leichter Anstieg im bürgerschaftlichen Engagement für ältere Menschen zu erkennen: Gaben die bürgerschaftlich Aktiven im Freiwilligensurvey des Jahres 1999 noch zu 8,2 Prozent an, primär für Ältere tätig zu sein, so lag dieser Wert zehn Jahre später bei 10,2 Prozent. Doch längst nicht jede dieser Tätigkeiten besteht in der Pflege oder Betreuung älterer Menschen. Eingrenzen lässt sich dies mithilfe des Tätigkeitsbereichs. Sehr wahrscheinlich sind Tätigkeiten, die der Pflege älterer Menschen entsprechen, im sozialen Bereich und im Bereich Gesundheit. In diesen Sektoren liegt zugleich der Anteil der Aktiven, die sich für ältere Menschen engagieren, weit über dem Durchschnitt (vgl. Tabelle 1): So richten Personen, die im sozialen Bereich tätig sind, ihre Tätigkeit zu 28,9 Prozent an älteren Menschen aus, der entsprechende Wert für den Gesundheitsbereich liegt bei 26,0 Prozent. Wird mittels dieser beiden Sektoren nun das Pflegevolumen geschätzt, das den Charakter bürgerschaftlichen Engagements aufweist, so entsprechen 5,5 Prozent aller bürgerschaftlichen Aktivitäten der Pflege älterer Menschen. Dieser Anteil ist seit 1999 sukzessive gestiegen und betrug in der ersten Erhebungswelle des Freiwilligensurvey (1999) noch 3,5 Prozent, fünf Jahre später 4,1 Prozent und heute (2009) 5,5 Prozent. Werden folglich unter dem Konzept bürgerschaftliches Engagement für Familien nicht nur Aktivitäten gefasst, die direkt Familien zugute kommen oder sich an Kinder und Jugendliche richten, sondern zählt man auch die Pflege und Betreuung älterer Menschen hinzu, so richten 35,2 Prozent aller bürgerschaftlich Engagierten ihr Angebot mittelbar oder unmittelbar an Familien.

Fragt man weiterhin nach dem durchschnittlichen zeitlichen Aufwand für die bürgerschaftliche Aktivität zeigt sich Folgendes: der geringste Zeitaufwand ergibt sich für jene Gruppe, die ein familienbezogenes bürgerschaftliches Engagement ausübt, das aber nicht einer Pflege- oder Betreuungsleistung für Ältere entspricht. Sie verwendet wöchentlich im Mittel 4,2 Stunden für die Ausübung ihrer Tätigkeit. Der entsprechende Wert für jene Befragten, die keiner familienbezogenen bürgerschaftlichen Aktivität nachgehen, beträgt 4,9 Stunden. Die meiste Zeit auf ihr Engagement verwenden Personen, die eine ältere Person pflegen oder betreuen. Ihr wöchentlicher Zeitaufwand beträgt im Mittel 5,2 Stunden. ${ }^{6}$

6 Dabei wurde die ordinalskalierte Variable zur Erfassung des Zeitaufwands in eine metrisch skalierte Variable überführt. Den Kategorien wurden die folgenden Werte zugewiesen: bis 2 Stunden (1), 3 bis 5 Stunden (4), 6 bis 10 Stunden (8), 11 bis 15 Stunden (13), über 15 Stunden (18); die Kategorie „,unregelmäßig“" wurde als fehlender Wert deklariert. 
Wechselt man nun die Perspektive und betrachtet die Geberseite des bürgerschaftlichen Engagements, so tritt mit Blick auf die Pflegeleistungen zugunsten älterer Menschen ein klarer Zusammenhang ans Licht: Die Betreuung und Pflege älterer Menschen wird vornehmlich von Älteren erbracht, wie die Abbildung 1 verdeutlicht:

Abbildung 1: Bürgerschaftliches Engagement in Form von Pflege- und Betreuungsleistungen in Abhängigkeit vom Alter des Aktiven

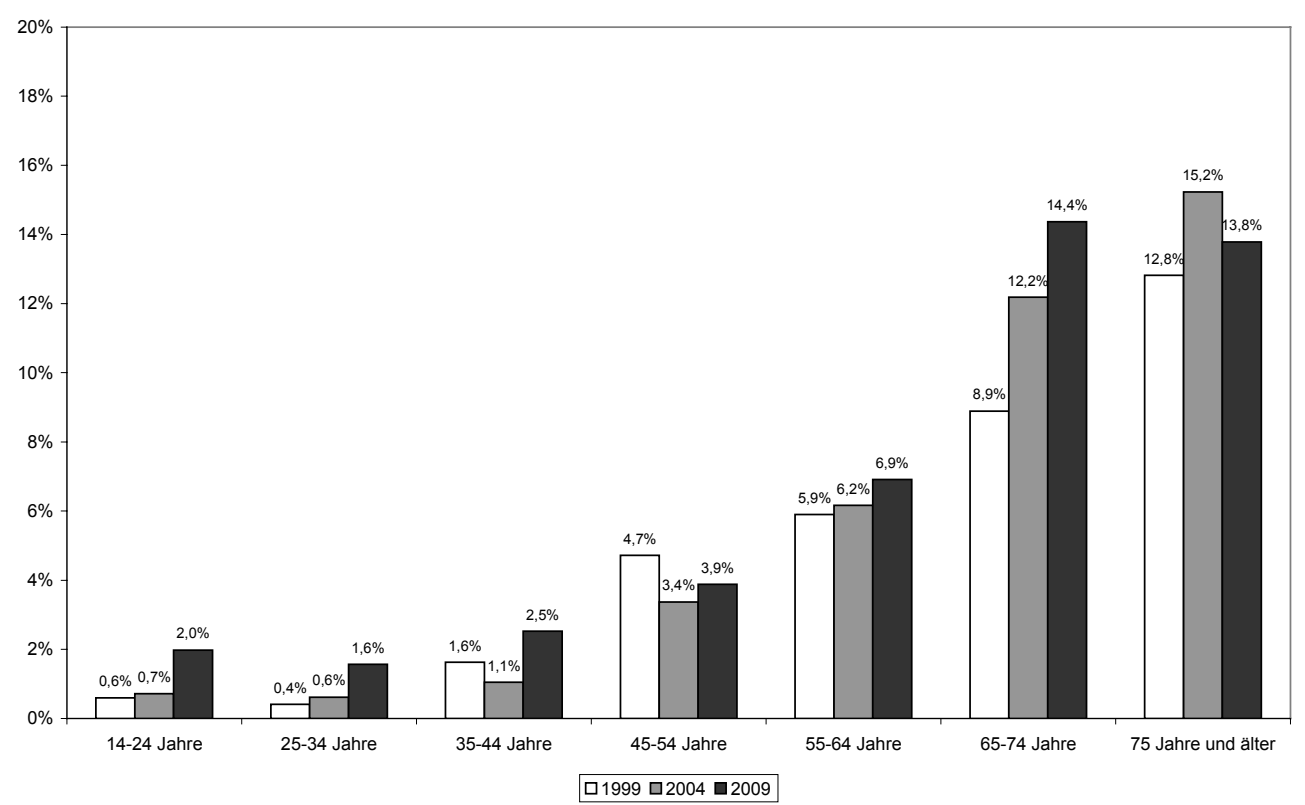

Quelle: Freiwilligensurvey 1999-2009; eigene Berechnungen; gewichtete Daten

Zwar wird für die beiden jüngsten Altersgruppen ein sehr geringer Anteil deutlich, der Pflege- und Betreuungsleistungen für ältere Menschen erbringt. Allerdings ist in diesen Kohorten ein überproportionaler Anstieg im Vergleich zur letzten Erhebungswelle von 2004 zu erkennen, der für die Zukunft hoffen lässt, dass das bereits praktizierte Prinzip „Alt hilft Alt“ durch das Prinzip „Jung hilft Alt“ zunehmend ergänzt wird.

\subsection{Bürgerschaftliches Engagement von Familien}

Wie ist es nun um das bürgerschaftliche Engagement von Familien bestellt? Um diese Frage zu beantworten werden zwei Analysewege beschritten: Der erste Weg betrachtet das familienbezogene bürgerschaftliche Engagement (inklusive der Pflege und Betreuung von älteren Menschen) von Personen mit minderjährigen Kindern im Haushalt in den Jahren 2004 und 2009. Dabei wird zusätzlich nach dem Alter des jüngsten Kindes differenziert (das Kleinkindalter 0 bis 2 Jahre; das Kindergartenalter zwischen 3 und 5 Jahren und das Schulalter zwischen 6 und 14 Jahren). Diese Unterscheidung ist der Annahme ge- 
schuldet, dass für diese Befragungspersonen unterschiedliche zeitliche Ressourcen zur Verfügung stehen, um einer bürgerschaftlichen Aktivität nachgehen zu können. Im Anschluss erfolgt eine detaillierte Analyse des familienbezogenen bürgerschaftlichen Engagements in Abhängigkeit von der Lebensform. Dabei werden drei Familienformen ebenfalls nach dem Lebensalter des jüngsten Kindes voneinander unterschieden. Neben dem Partnerhaushalt mit Kind werden als Vergleichsgruppen Alleinerziehende, Paare ohne eigene Kinder im Haushalt und Alleinstehende betrachtet. Für die Gruppe der Alleinerziehenden ist eine weitere Differenzierung nach dem Alter des Kindes nicht möglich, da sonst die Fallzahlen zu gering ausfallen. Daher werden die Alleinerziehenden mit mindestens einem Kind im Vorschul- oder Schulalter betrachtet. Paare ohne Kinder im Haushalt als auch die Alleinstehenden werden dagegen nach dem Alter der Befragungsperson differenziert. Hier ist das Kriterium, ob die befragte Person, die in der entsprechenden Lebensform lebt, jünger oder älter als 50 Jahre ist. Diese detaillierte Analyse zum Zusammenhang zwischen Lebensform und bürgerschaftlichem Engagement bezieht sich auf den Freiwilligensurvey 2004, da dieser, im Unterschied zur Erhebungswelle 2009, nähere Informationen zur Haushaltszusammensetzung enthält.

Tabelle 2: Bürgerschaftliches Engagement und familienbezogenes Engagement in Abhängigkeit vom Alter des jüngsten Kindes

\begin{tabular}{lcccc}
\hline & \multicolumn{2}{c}{$\mathbf{2 0 0 4}$} & 2009 \\
& $\begin{array}{c}\text { Anteil der ehrenamt- davon: für Familien } \\
\text { lich Aktiven }\end{array}$ & $\begin{array}{c}\text { Anteil der ehrenamt- davon: für Familien } \\
\text { engagiert }\end{array}$ & $\begin{array}{c}\text { lich Aktiven } \\
\text { engagiert }\end{array}$ \\
\hline alle Befragten & $35,7 \%$ & $33,7 \%$ & $35,9 \%$ & $35,2 \%$ \\
jüngstes Kind 0-2 Jahre & $34,9 \%$ & $43,4 \%$ & $35,1 \%$ & $47,4 \%$ \\
jüngstes Kind 3-5 Jahre & $42,6 \%$ & $54,1 \%$ & $41,6 \%$ & $67,2 \%$ \\
jüngstes Kind 6-14 Jahre & $46,1 \%$ & $45,4 \%$ & $46,6 \%$ & $50,0 \%$ \\
kein Kind in diesen Altersgruppen & $33,8 \%$ & $29,2 \%$ & $34,0 \%$ & $29,0 \%$ \\
\hline
\end{tabular}

Quelle: Freiwilligensurvey 2004-2009; eigene Berechnungen; gewichtete Daten

Betrachtet man zunächst die allgemeinen Engagementquoten, so weisen Personen mit Vorschul- oder Schulkindern im Haushalt ein überdurchschnittliches Engagement auf. Insbesondere stechen die hohen Quoten für Eltern mit Schulkindern hervor. Leben Kinder im Haushalt, die zwischen 0 und 2 Jahre alt sind, so liegt die Engagementquote nur leicht unter dem Gesamtmittelwert. Dieser Verlauf der Engagementquote bestätigt die Vermutung, wonach das Engagement von Eltern in einem engen Zusammenhang mit dem Betreuungsbedarf der eigenen Kinder steht. Zudem wird deutlich, dass Befragte, die nicht mit einem unter 15-jährigen Kind in einem Haushalt leben, in beiden Erhebungswellen eine um rund zwei Prozentpunkte geringere Engagementquote aufweisen als die jeweilige Gesamtstichprobe.

Deutlich wird zudem, dass familienbezogenes bürgerschaftliches Engagement durch das Vorhandensein von Kindern im Haushalt maßgeblich begünstigt wird. Besonders in der Gruppe der Personen mit Kindern im Vorschulalter (3-5 Jahre) sind die Werte beträchtlich: 2009 engagierten sich unter allen bürgerschaftlich Aktiven, deren jüngstes Kind sich im Vorschulalter befindet, rund zwei von drei Befragten (67,2 Prozent) für Familien. Dagegen gestaltet sich das bürgerschaftliche Engagement für Familien unter- 
durchschnittlich, wenn keine Kinder unter 15 Jahren im Haushalt leben. Schließlich hat sich der positive Zusammenhang zwischen familienbezogenem Engagement und dem Vorhandensein von Kindern im Haushalt gegenüber 2004 in allen Kategorien verstärkt.

Abschließend soll nun das familienbezogene bürgerschaftliche Engagement in Abhängigkeit von der praktizierten Lebensform anhand der Erhebungswelle von 2004 untersucht werden. Hierüber gibt die Abbildung 2 Auskunft:

Abbildung 2: Bürgerschaftliches Engagement und familienbezogenes Engagement in Abhängigkeit von der Lebensform

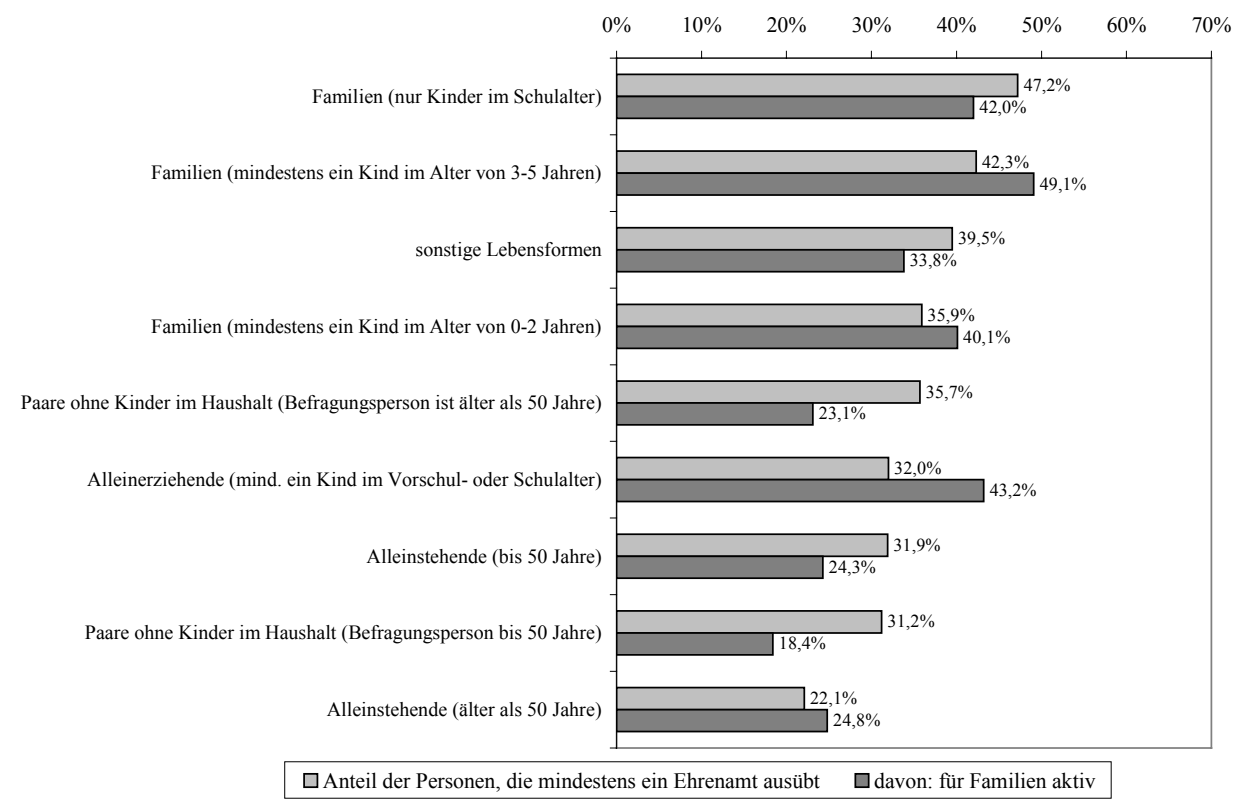

Quelle: Freiwilligensurvey 2004; eigene Berechnungen; gewichtete Daten

Familien sind demnach nicht nur überproportional häufig bürgerschaftlich engagiert, ihre Aktivität kommt auch zu großen Anteilen wiederum Familien zugute. Von allen Personen, die in familiären Kontexten leben und sich bürgerschaftlich engagieren, profitieren in mindestens 40 Prozent dieser Fälle mittelbar oder unmittelbar andere Familien von diesen Aktivitäten. Der höchste Wert findet sich dabei in der Gruppe der Familien mit mindestens einem Kind im Kindergartenalter (49,1 Prozent), während unter den Befragungspersonen mit Kleinkindern im Haushalt die (familienbezogene) Engagementquote etwas niedriger ausfällt (40,1 Prozent). Beachtlich ist zudem das Engagement von Alleinerziehenden: Zwar ist ihre Engagementquote mit knapp einem Drittel unterdurchschnittlich; die Aktiven unter ihnen richten ihre Tätigkeit aber in 43,2 Prozent aller Fälle an Familien. Wird das angespannte Zeitregime vieler Ein-Eltern-Familien bedacht (Schneider 2008), so verweist dieses relativ hohe bürgerschaftliche Engagement der Alleinerziehenden nicht nur auf einen hohen Grad an Gemeinsinn, sondern reflektiert wohl auch die Schwierigkeit auf dem regulären Arbeitsmarkt Fuß zu fassen sowie den Wunsch über bürgerschaftliches 
Engagement Anerkennung und Teilhabe zu erreichen. In der Zusammenschau zeigt sich folgendes Bild: Familien weisen ein überdurchschnittlich hohes bürgerschaftliches Engagement auf. Insgesamt ist also nicht nur bürgerschaftliches Engagement für Familien weit verbreitet. Gleiches gilt auch für bürgerschaftliches Engagement von Familien und noch stärker für bürgerschaftliches Engagement von Familien, das wiederum für andere Familien bestimmt ist.

\section{Zusammenfassung und Perspektiven}

Familien profitieren, das zeigen die Daten des Freiwilligensurvey von 1999 bis 2009, von bürgerschaftlichem Engagement, insbesondere durch Betreuungsleistungen, die sich an Kinder/Jugendliche oder Senioren richten. So übten im Jahre 2009 knapp 36 Prozent der über 14-Jährigen ein bürgerschaftliches Engagement aus. Von diesen Aktiven richteten 35 Prozent ihre Tätigkeit direkt oder indirekt an Familien. Familienmitglieder gehören gleichzeitig aber auch zu den Hauptakteuren der bürgerschaftlichen Arbeit, insbesondere der bürgerschaftlichen Arbeit für (andere) Familien.

Eine hohe Engagementquote spricht für eine lebendige Gemeinschaft und eine funktionierende Zivilgesellschaft. Insbesondere die konstant hohen Engagementquoten der jüngeren Generation bestätigen im Zusammenspiel mit der gewachsenen Bereitschaft, soziales Engagement auch verstärkt in Richtung der älteren Generation (Pflege- und Betreuungsbedarf) zu geben, dieses Bild (vgl. auch die Shell Jugendstudie 2010). Die aktuelle gesellschaftliche Diskussion über ein freiwilliges soziales Jahr zeigt zudem die hohe Akzeptanz sozialen Engagements und zwar sowohl in der Politik als auch in der Bevölkerung.

Die Orientierung bürgerschaftlichen Engagements auf Leistungen für die Familie passt auch zum aktuellen familienpolitischen Diskurs. Dort wird leidenschaftlich nach Wegen gesucht, die Familien zu unterstützen. Dieser Diskurs ist naturgemäß stark mit Blick auf die Bedarfe der bedürftigen Familien geführt. Als zentrale Problemlage heutiger Familien muss die finanziell angespannte Situation (Armut) vieler Familien angesehen werden. Dies wirkt sich insbesondere auf die Bildungschancen und die Gesundheitsbiographien der Kinder aus. Hier kann eine Förderung bürgerschaftlichen Engagements zur Stärkung der Vernetzung der Familien (soziales Kapital) beitragen und so bei der Bewältigung finanzieller Knappheit helfen. Bürgerschaftliches Engagement mag aber auch für die von Armut betroffenen Jugendlichen ein Weg sein, der eigenen Lebenswelt zu entkommen. Die geringen Chancen benachteiligter Jugendlicher auf dem ersten Arbeitsmarkt können über bürgerschaftliches Engagement und die so gewonnenen Erfahrungen und das dort gezeigte Engagement gesteigert werden. Leider muss aber auch hier konstatiert werden: die Bereitschaft für soziales Engagement ist wie in vielen anderen Lebensbereichen sozial ungleich verteilt: Je besser der familiäre Hintergrund, desto stärker ist die Bereitschaft für bürgerschaftliches Engagement ausgeprägt. Aber gerade deshalb sollte dieser Weg gefördert werden, stellt er doch den Zusammenhang von bürgerschaftlichem Engagement und Familie, wie er hier betrachtet wurde, noch einmal in eine andere Perspektive. Nämlich die Möglichkeit, dass junge Menschen durch bürgerschaftliches Engagement, Wege aus der Armut beschreiten. 
Weiterhin ist vor dem Hintergrund des demografischen Wandels mit einem zunehmenden Bedarf an bürgerschaftlicher Unterstützung bei der Betreuung und Pflege älterer Menschen zu rechnen. Die Daten des Freiwilligensurvey lassen einen steigenden Anteil an Befragten erkennen, der sich in diesem Tätigkeitsfeld engagiert. Waren es in der Vergangenheit fast ausschließlich Ältere, die in der Betreuung und Pflege älterer Menschen ehrenamtlich aktiv waren, so ist im neuen Freiwilligensurvey 2009 ein überproportionaler Anstieg in den jüngeren Alterskohorten zu verzeichnen. Somit erscheint es für die $\mathrm{Zu}-$ kunft denkbar, dass das bereits praktizierte Prinzip „Alt hilft Alt“ durch das Prinzip „Jung hilft Alt" zunehmend ergänzt wird.

Werfen wir selbstkritisch einen Blick auf die Forschungslage zum freiwilligen Engagement im Familienkontext, so fällt insbesondere auf, dass es keine gesicherten Erkenntnisse darüber gibt, welcher Bedarf (Art und Umfang) an Engagement aus Sicht der Familien vorhanden ist (eine Bedarfsanalyse). Ebenso gibt es keine Erkenntnis darüber, wie die schon geleistete freiwillige Arbeit für Familien von diesen im Hinblick auf Qualität und Umfang bewertet wird (eine Zufriedenheitsanalyse). Beide Aspekte würden nicht nur der Forschung, sondern auch den Freiwilligenagenturen und Familienverbänden wertvolle Hinweise liefern können.

\section{Literatur}

Alscher, M.. Dathe, D., Priller, E. \& Speth, R. (2009). Bericht zur Lage und zu den Perspektiven des bürgerschaftlichen Engagements in Deutschland. Projektgruppe Zivilengagement Wissenschaftszentrum Berlin für Sozialforschung. Berlin: WZB (Herausgegeben vom Bundesministerium für Familie, Senioren, Frauen und Jugend. Berlin).

Bundesministerium für Familien, Senioren, Frauen und Jugend (2006). Siebter Familienbericht. Familie zwischen Flexibilität und Verlässlichkeit. Perspektiven für eine lebenslaufbezogene Familienpolitik. Berlin: BFSFJ.

Corsten, M. \& Kauppert, M. (2007). Wir-Sinn und fokussierte Motive. Zur biografischen Genese bürgerschaftlichen Engagements. Zeitschrift für Soziologie, 36, 5, S. 346-363.

Geiss, S. \& Picot, S. (2009). Familien und Zeit für freiwilliges Engagement. In: M. Heitkötter, K. Jurczyk, A. Lange \& U. Meier-Gräwe (Hrsg.), Zeit für Beziehungen? Zeit und Zeitpolitik für Familien. Opladen \& Farmington Hills, MI: Verlag Barbara Budrich, S. 291-317.

Gensicke, T. (2005a). Freiwilliges Engagement in Deutschland 1999-2004. Kurzfassung. München: TNS Infratest Sozialforschung.

Gensicke, T. (2005b). Freiwilliges Engagement in Einrichtungen für ältere Menschen, Kinder und Jugendliche sowie für Menschen mit Behinderung. Sonderauswertung für die Bundesarbeitsgemeinschaft der Freien Wohlfahrtspflege auf Basis des Freiwilligensurveys 2004. München: TNS Infratest Sozialforschung.

Gensicke, T., Picot, S. \& Geiss, S. (2006). Freiwilliges Engagement in Deutschland 1999-2004. Wiesbaden: VS Verlag für Sozialwissenschaften.

Gensicke, T. \& Geiss, S. (2010). Hauptbericht des Freiwilligensurvey 2009. Berlin: BFSFJ (Herausgegeben vom Bundesministerium für Familie, Senioren, Frauen und Jugend).

Heitkötter, M. \& Jurczyk, K. (2009). Freiwilliges Engagement von und für Familien - politische Rahmungen. In: T. Olk, A. Klein \& B. Hartnuß (Hrsg.), Engagementpolitik. Die Entwicklung der Zivilgesellschaft als politische Aufgabe. Wiesbaden: VS Verlag für Sozialwissenschaften, S. 491-509.

Kaufmann, F.-X. (1995). Zukunft der Familie. München: C.H. Beck.

Klocke, A., Limmer, R. \& Lück, D. (2001). Das Ehrenamt im Umfeld der Familie. Bamberg: Staatsinstitut für Familienforschung an der Universität Bamberg (ifb-Materialien 7/01). 
Klocke, A. \& Stadtmüller, S. (2009). Wandel der Familienformen in Deutschland und die Bedeutsamkeit des bürgerschaftlichen Engagements. Frankfurt am Main: Forschungszentrum demografischer Wandel, Fachhochschule Frankfurt am Main (FZDW Bericht Nr. 3), http://www.fh-frankfurt.de/de/. media/fzdw/wandel_der_familienformen.pdf.

Klocke, A. \& Stadtmüller, S. (2010). Demografische und sozioökonomische Trends im Jugendalter und deren Auswirkungen auf das bürgerschaftliche Engagement. BBE-newsletter Nr. 4/2010.

Priller, E. (2004). Konkurrierende Konzepte zum bürgerschaftlichen Engagement in der Langzeitperspektive. Forschungsjournal Neue Soziale Bewegungen, Zwischen Meier und Verein - Modernisierungspotentiale im Ehrenamt, 1/2004, S. 36-43.

Schneider, N. (Hrsg.) (2008). Lehrbuch Moderne Familiensoziologie. Opladen \& Farmington Hills, MI: Verlag Barbara Budrich (UTB).

Shell Deutschland (Hrsg.) (2010). 16. Shell Jugendstudie 2010. Frankfurt am Main: Fischer Verlag.

Statistisches Bundesamt (Hrsg.) (2008). Datenreport 2008. Zahlen und Fakten für die Bundesrepublik Deutschland. Bonn: Statistisches Bundesamt.

Eingereicht am/submitted on: 16.03.2010

Angenommen am/accepted on: 10.01.2011

Anschriften der Autoren/Addresses of the authors:

Prof. Dr. Andreas Klocke (Korrespondenzautor/corresponding author)

Sven Stadtmüller, M.A.

Forschungszentrum Demografischer Wandel (FZDW)

Fachhochschule Frankfurt am Main

Nibelungenplatz 1

60318 Frankfurt am Main

E-Mail: aklocke@ffzdw.fh-frankfurt.de svenstad@fzdw.fh-frankfurt.de 